\title{
Evaluation of MRI T2-signal intensities of GH-secreting pituitary macroadenoma in treatment-naïve acromegalic patients receiving primary treatment with lanreotide Autogel $120 \mathrm{mg}$
}

Philippe Caron, ${ }^{1}$ Louis-David Rivière, ${ }^{2}$ Aude Houchard ${ }^{3}$ Caroline Sert, ${ }^{3}$ Fabrice Bonneville, ${ }^{2}$ on behalf of the PRIMARYS study group

${ }^{4}$ Dept of Endocrinology \& Metabolic Diseases, CHU Larrey, Toulouse, France; ${ }^{2}$ Dept of Neuroradiology, CHU Purpan, Toulouse, France; ${ }^{3} / p s e n$, Boulogne Billanco

\section{Introduction and objectives}

\section{Methods}

SR, signal ratio

Acromegaly is a chronic condition characterized by excess growt hormone (GH) secretion, which is caused, in the majority of cases, by a pituitary adenoma. ${ }^{1}$ Long-acting somatostatin analogues (SSAs) are well established treatments for acromegaly after unsuccessful surgery, and are also used as first line treatment if surgery is refused, contra-indicated or unlikely to be successful.

In a previous study, the hypointense T2-signal of GH-secreting pituitary adenomas on magnetic resonance imaging (MRI), whic reflects various tissue properties, predicted the biochemical outcome of first-line SSA therapy.

However, the definition of $\mathrm{T} 2$ hypointensity of GH-secreting adenomas varies between studies. We hypothesize that different definitions of T2 hypo-, iso- and hyperintensities lead to differences in perceived distributions of GH-secreting adenomas, and therefore to differences in reported outcomes after SSA treatment.

Here, we investigate three methods for evaluating the T2. signal intensity of pituitary macroadenoma, and the extent o which they predict the response to SSA treatment, using additional post hoc analysis of data from the PRIMARYS study (EudraCT2007-000155-34; NCT00690898).

- PRIMARYS was an open-label study in which 90 patients with acromegaly received primary medical treatment with the longacting SSA, lanreotide Autogel (Depot in the USA) at a fixed dose of $120 \mathrm{mg}$ every 4 weeks for 1 year. The study was conducted to evaluate the tumour volume reduction in patients with macroadenomas (diameter $\geq 10 \mathrm{~mm}$ ) via centralized MRI readings. Patients were eligible for inclusion if they were treatment-naïve and had no visual field defects.

In the current analysis, each MRI was read by a single neuroradiologist to determine T2-signal intensity, using one qualitative method based on a visual assessment (as per daily routine practice) and two quantitative methods, the Heck method ${ }^{3}$ and a method using the signal intensity ratio of the adenoma under the grey matter (Figure 1)

For each method, intensities were rated as hypointense, isointense or hyperintense, as defined in Figure 1. The reader was blinded to the identity of the subject and the status of hormonal control and tumour response.

For each of the three methods, signal intensities at baseline were summarized in the overall population and according to hormona control and tumour response (endpoints of the PRIMARYS study4). Hormonal control was defined as GH levels $\leq 2.5 \mathrm{ng} / \mathrm{mL}$ and normalized insulin-like growth factor-1 (IGF-1).

Tumour response was defined as a reduction in tumour volume $\geq 20 \%$ between baseline and the patient's last visit during the study.

Multivariate analyses were conducted to evaluate whether, after controlling for other baseline characteristics, baseline T2-signal intensities were associated with the change in $\mathrm{GH}$ and IGF-1, hormonal control and tumour response at last study visit available (LVA).

Figure 1. Methods used to assess and classify T2-signal intensities of macroadenomas in patients with acromegaly
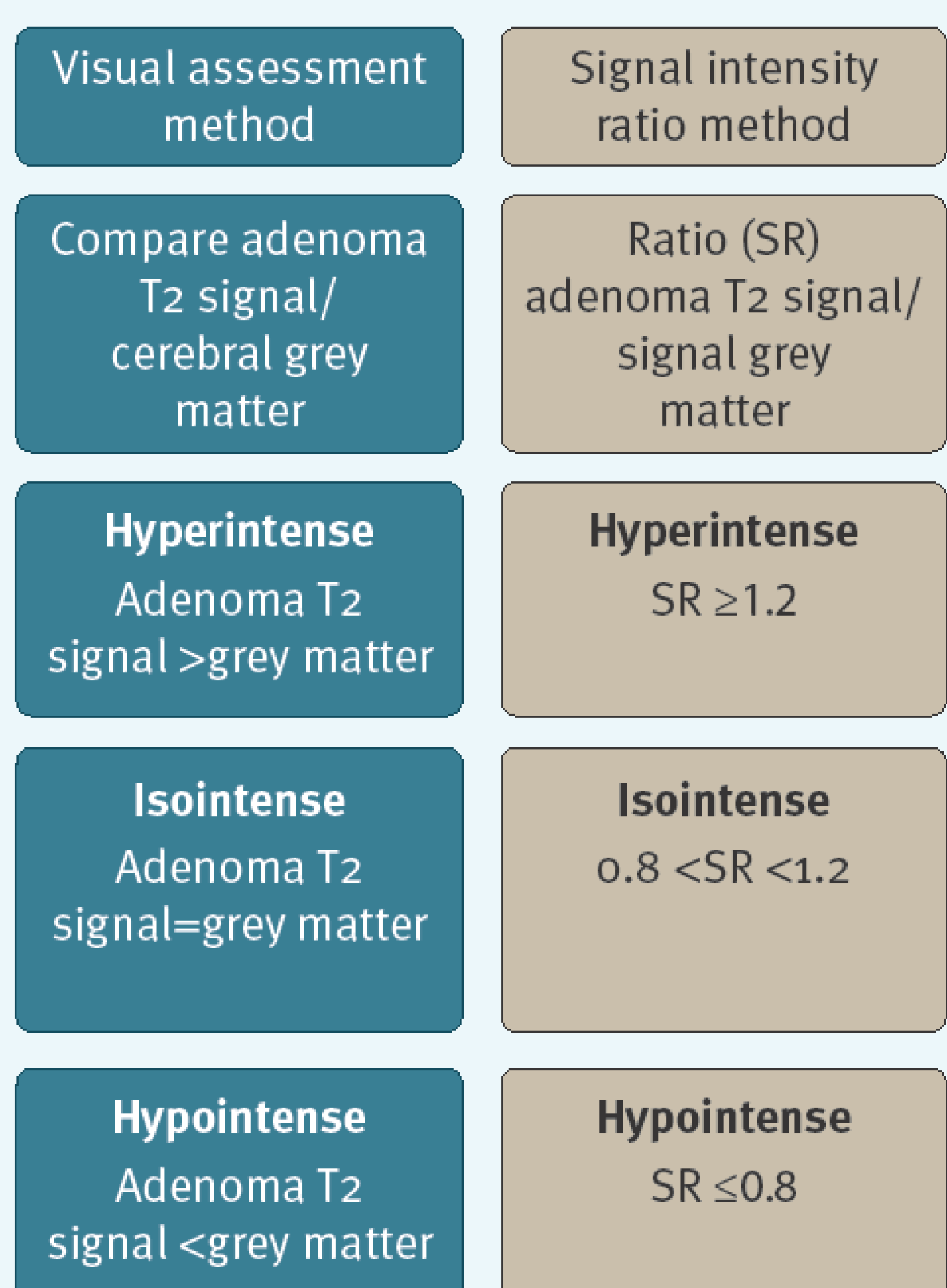

$\mathrm{SR} \leq 0.8$

Adenoma T2
signal $<$ grey matter

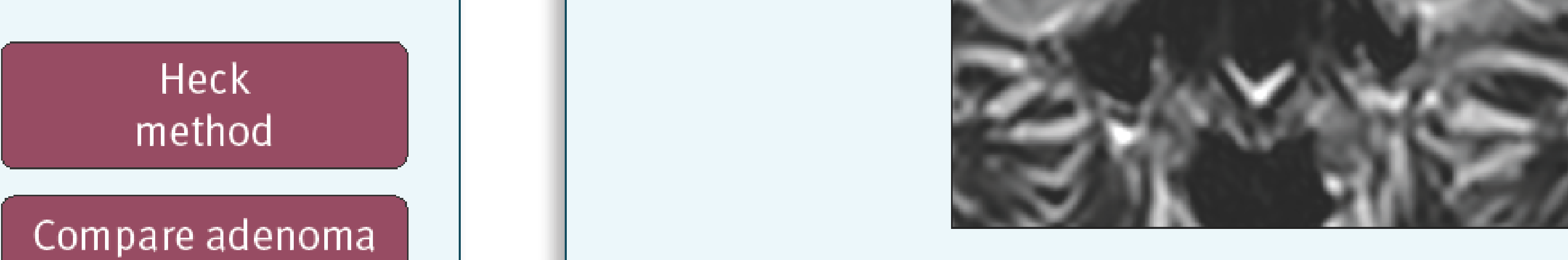

(b) Quantitative analysis

Hypointense

Adenoma T2 signal <white matter signal grey matter
AND white matter Hyperintense Adenoma T2

Isointense Adenoma $\mathrm{T}_{2}$ and white matter
Results

Baseline 22 signal intensity data were available for 85 patients, esponse at LVA.

Overall, more adenomas were classified as hypointense using Ovis 20\%) (Table 1)

Figure 2 shows an MRI scan of a patient classified as hypointense using both the visual assessment and the signal-ratio methods, but isointense using the

Patients achieving hormonal control or tumour response were more often classified as hypointense according to the visual and Figure 4).

- As targeted adenomas appear to be hypointense on

T2-weighted images, we arbitrarily selected the visua

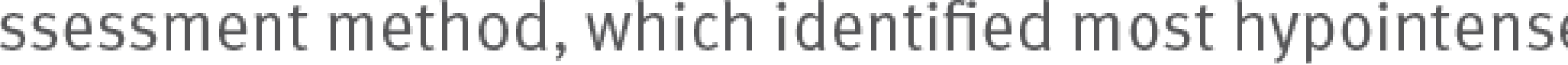
adenomas, for further analyses.

Baseline tumour volumes were lower in the hypointense grou than in the hyperintense and isointense groups: median (95\% CD and $2017(1387 ; 4066) \mathrm{mm}^{3}$, respectively.

- There was an additional reduction in $\mathrm{GH}$ levels of $4 \mu \mathrm{g} / \mathrm{L}$ between baseline and LVA for those with hypointense versus isointense tumours ( $p<0.0001$, F-test).

Similarly, there was an additional reduction in IGF-1 levels (of versus isointense tumours ( $p=0.0026$, F-test). 列

Table 1. Proportions of patients classified as hypointense, isointense and hyperintense according to T2-signal intensities

\begin{tabular}{lccc|} 
& $\begin{array}{c}\text { Visual } \\
\text { assessment }\end{array}$ & $\begin{array}{c}\text { Signal-ratio } \\
\text { method }\end{array}$ & Heck method \\
\hline Hypointense, $\mathrm{n}(\%)$ & $50(59)$ & $31(36)$ & $17(20)$ \\
Isointense, $\mathrm{n}(\%)$ & $31(36)$ & $44(52)$ & $40(47)$ \\
Hyperintense, $\mathrm{n}(\%)$ & $4(5)$ & $10(12)$ & $28(33)$ \\
\hline
\end{tabular}

Figure 2. Illustrative example of a GH-secreting pituitary macroadenoma at baseline on MRI (coronal T2-weighted sequence) (a) Qualitative (visual) analysis

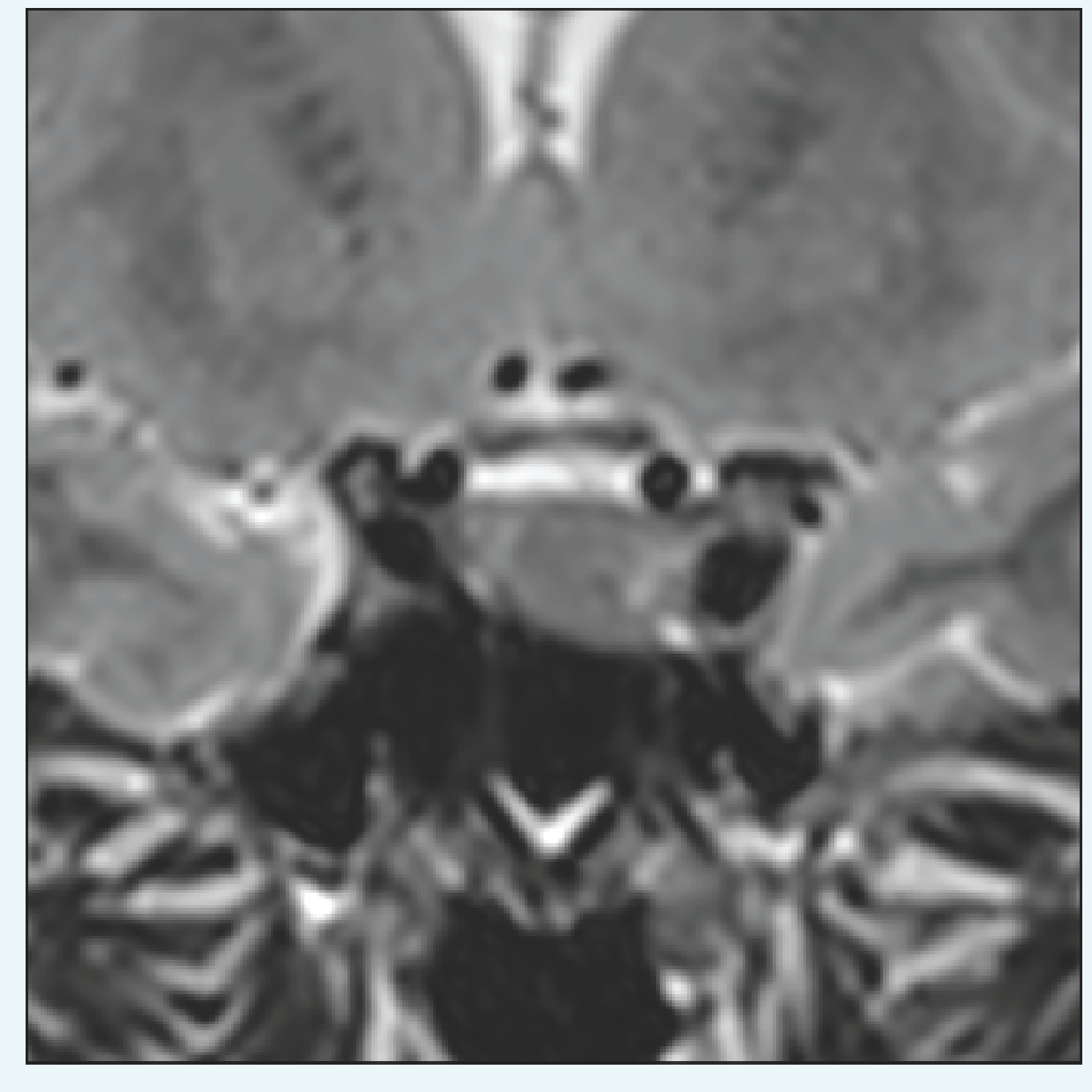
signal > grey matter

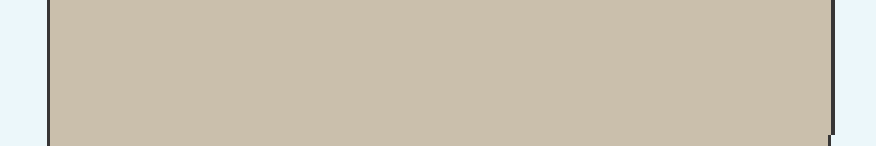

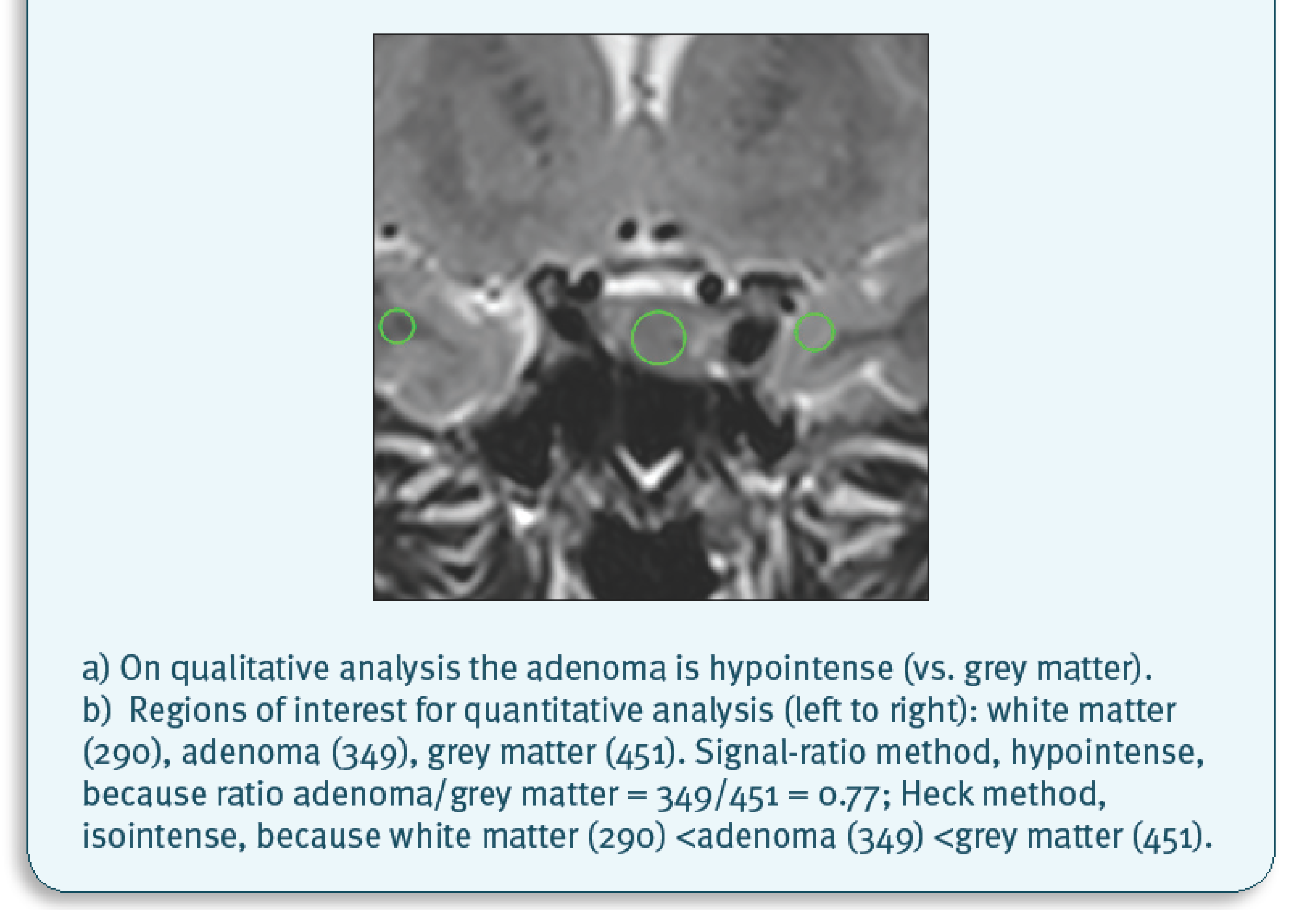

Figure 3. Hormonal control according to baseline MRI T2 signal intensity using visual assessment, signal ratio and Heck methods

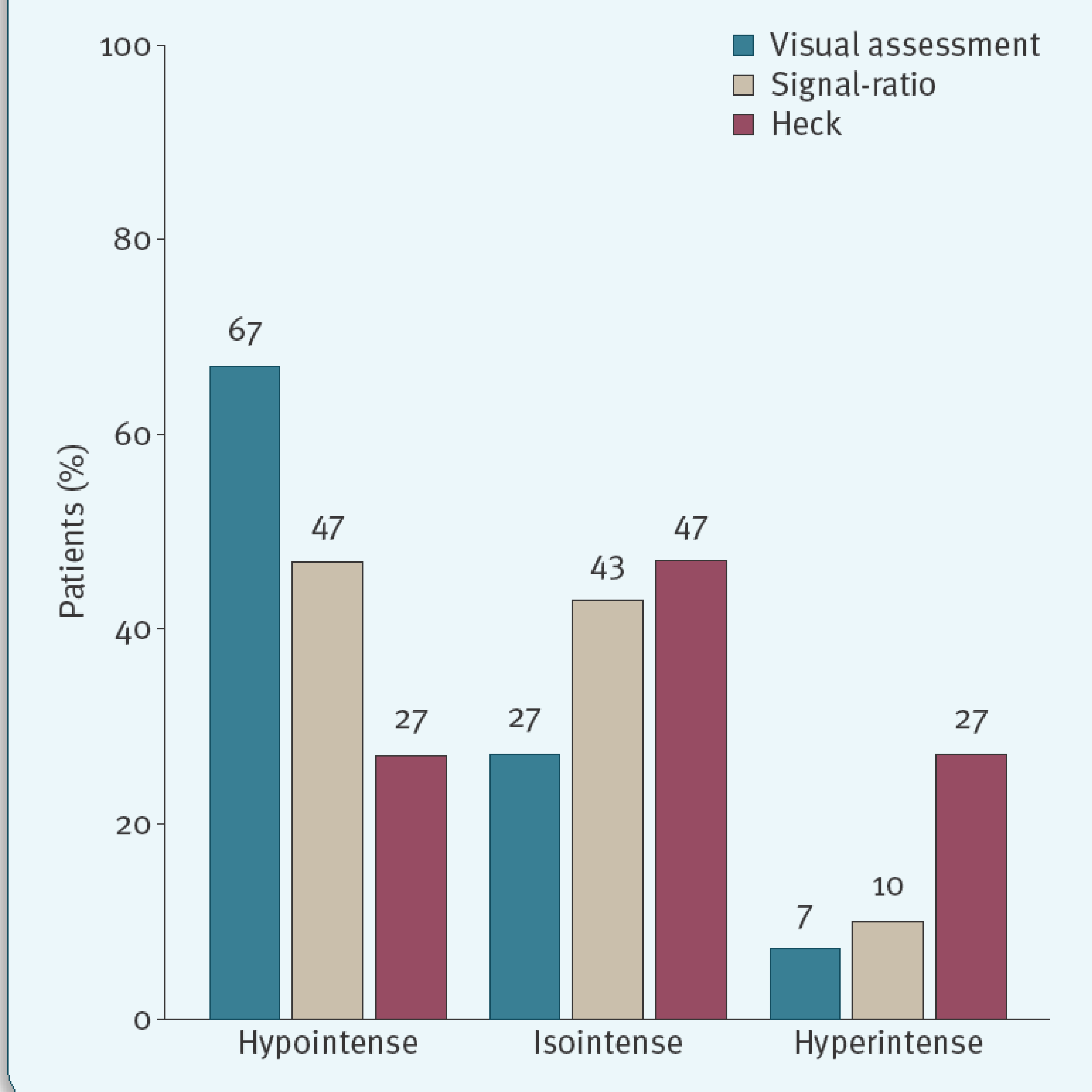

Figure 4. Tumour response according to baseline MRI T2-signal intensity using visual assessment, signal ratio and Heck methods

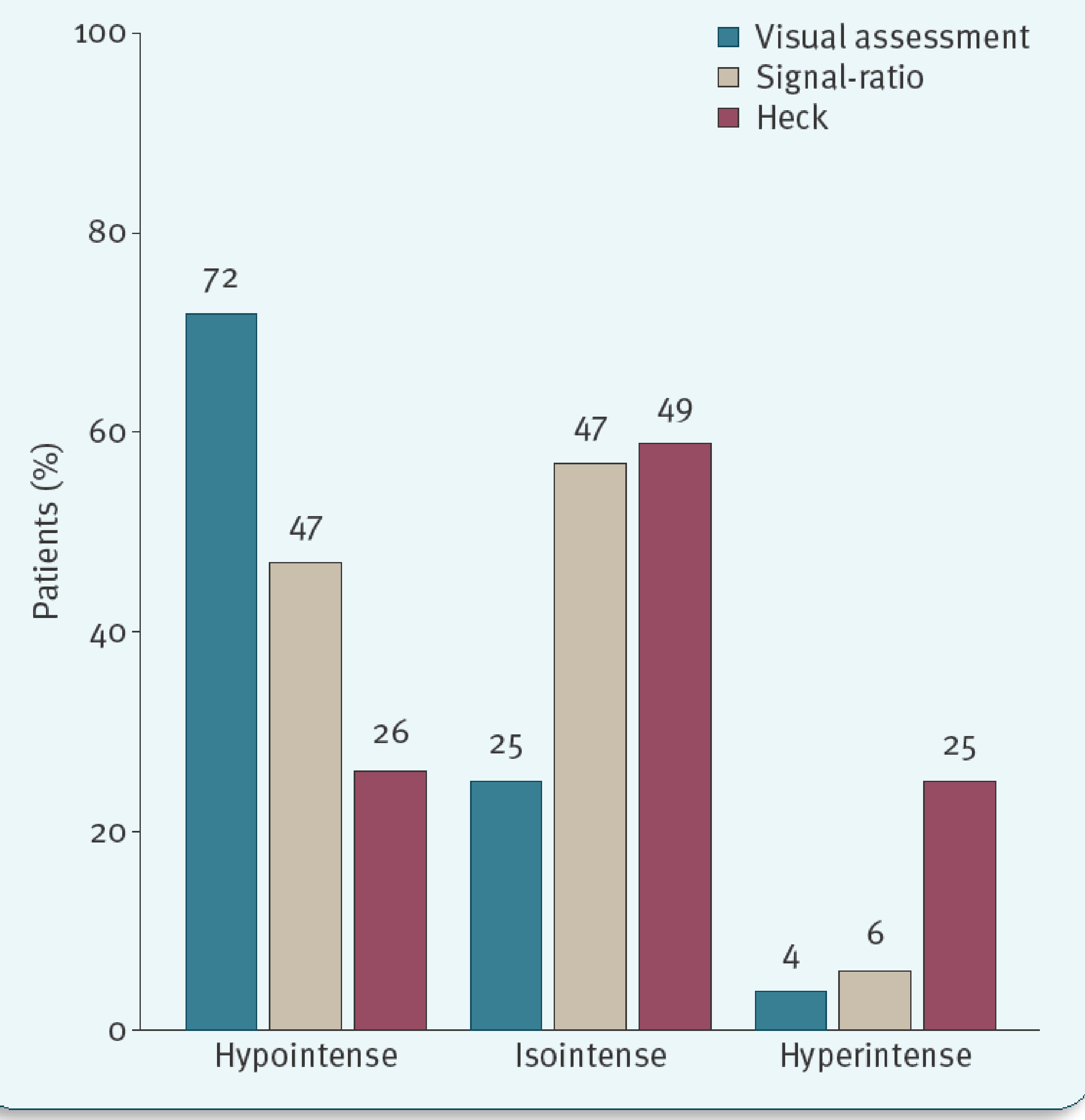

Conclusions

- These results suggest that clinical visual assessment is preferable for the identification of T2-hypointense, GH-secreting macroadenoma in treatment-naïve patients with acromegaly.

- There was a trend towards smaller tumour volumes at baseline in the hypointense group.

- Patients with hypointense lesions have a greater reduction in $\mathrm{GH}$ and IGF-1 values compared with those with isointense lesions following primary SSA treatment. In addition, the odds of tumour response are higher.

PRIMARYS Study Group

Belgium: L. Van Gaal; Czech Republic: I. Marek; Finland: P. Nuutila, M. Välimäk France: C. Aizenberg. F. Borson-Chazot, T. Brue, P. Caron, O. Chabre, P. Chanson, C. Cortet Rudelli, B. Delemer, J.-M. Kuhn, A. Tabarin; Germany: K. Badenhoop, C. Berg, S. Petersenn, C. Schöfl, I. Schopohl; Italy: S. Cannavô, A. Colao, . De Marinis; the Netherlands: A. Stades, A.J. van der Lely; Turkey P. Kadıoğlu; UK: J.S. Bevan, D. Flanagan, P. Trainer

References

(ina C, WasS JAH.J Endocrinol 2015,226:1141-60.

Heck A, et al Clin Endocrinol 2012:77:72-8.

Clin Endocrinol Metab 2014: 